\title{
Unsatisfactory outcomes in myasthenia gravis: influence by care providers
}

\author{
Murielle Dunand · Stephan A. Botez $\cdot$ \\ François-Xavier Borruat · Pascale Roux-Lombard • \\ François Spertini · Thierry Kuntzer
}

Received: 8 July 2009/Revised: 28 August 2009/Accepted: 1 September 2009/Published online: 17 September 2009

(C) Springer-Verlag 2009

\begin{abstract}
Myasthenia gravis (MG) can be difficult to treat despite an available therapeutic armamentarium. Our aim was to analyze the factors leading to unsatisfactory outcome (UO). To this end we used the Myasthenia Gravis Foundation of America classification system. Forty one patients with autoimmune MG were followed prospectively from January 2003 to December 2007. Outcomes were assessed throughout follow-up and at a final visit. 'Unchanged', 'worse', 'exacerbation' and 'died of MG' post-intervention status were considered UOs. During follow-up, UO rates reached $54 \%$ and were related to undertreatment $(41 \%)$, poor treatment compliance $(23 \%)$, infections (23\%), and adverse drug effects (13\%). The UO rate at final study assessment was $20 \%$. UO during followup was significantly $(P=0.004)$ predictive of UOs at final assessment. When care was provided by neuromuscular (NM) specialists, patients had significantly better follow-up
\end{abstract}

M. Dunand $(\bowtie) \cdot$ S. A. Botez $\cdot$ T. Kuntzer

Nerve-Muscle Unit, Neurology Service,

Centre Hospitalier Universitaire Vaudois and University

of Lausanne, 1011 Lausanne, Switzerland

e-mail: murielle.dunand@chuv.ch

F.-X. Borruat

Neuro-Ophtalmology Unit, Jules Gonin Hospital,

Centre Hospitalier Universitaire Vaudois,

University of Lausanne, Lausanne, Switzerland

P. Roux-Lombard

Immunology and Allergy Service,

Geneva University Hospital (HUG),

Geneva, Switzerland

F. Spertini

Division of Immunology and Allergy,

Centre Hospitalier Universitaire Vaudois,

University of Lausanne, Lausanne, Switzerland scores $(P=0.01)$. At final assessment UO rates were $7 \%$ and significantly better in patients treated by NM specialists, compared to other physicians where UO rates reached $27 \%$. UO was a frequent finding occurring in more than half our patients during follow-up. Nearly two-thirds of the UOs could have been prevented by appropriate therapeutic adjustments and improved compliance. The differential UO rates at follow-up, their dependency on the degree to which the management was specialized and their correlation with final outcomes suggest that specialized MG care improves outcomes.

Keywords Myasthenia gravis - Outcome - MGFA score · Care provider · Treatment

\section{Introduction}

Fluctuating muscle weakness, with or without remission or exacerbation, is characteristic of myasthenia gravis (MG), an autoimmune disease of the postsynaptic neuromuscular (NM) junction. Disease modifying immunotherapy aims to achieve complete, or near complete, remission within a few months $[10,12]$ allowing patients to resume their daily activities in full. This response has to be maintained and exacerbation prevented, but in practice this can be difficult to achieve. We report the results of a prospective study performed to assess the rate and causes of unsatisfactory outcomes (UOs).

\section{Methods}

We conducted a prospective non-randomised observational study from January 2003 to December 2007. A 36 item 
database was constructed to facilitate analysis of demographic, clinical, electrophysiological, pharmacological, immunological, surgical, histological and therapeutic data.

\section{Patients}

All patients examined at our hospital were included consecutively, provided they had (1) a clinical picture compatible with MG, and (2) at least one of the following abnormal tests: a significant decrement in the evoked compound motor action potentials $(>10 \%)$ after repetitive nerve stimulation (RNS) of at least two nerves, abnormal jitter or blocking on single fiber electromyography [1]; positive anti-acetylcholine receptor (AChR) antibodies with a titer greater than $0.5 \mathrm{nmol} / \mathrm{l}$ or anti-muscle-specific tyrosine kinase (MuSK) antibodies at greater than $0.05 \mathrm{nmol} / \mathrm{l}$. The same neurologist from the neuromuscular treatment center examined all patients at diagnosis and identified patients for inclusion in the study. Follow-up examinations were then performed by either neurologists with NM subspecialty training (NM neurologists), or by other hospital or primary care neurologists. NM neurologists had the Swiss board certification in neurology and in neurophysiology and had a special training at the Institute of Myology in Paris. The follow-up mode was determined by patient choice (as allowed by the Swiss insurance system) or referral mode (hospitalized patients in different units versus those attending outpatient clinics); subsequently, the patients were divided in two groups, one in which patients were under the care of NM neurologists, and a second in which the patients received treatment from other neurologists (Fig. 1).

Clinical evaluation and outcome measures

Patients were scored according to the Myasthenia Gravis Foundation of America (MGFA) classification system [6]. Assessments were performed at least three times by the NM neurologist: at the 'diagnostic visit', 'during followup' (which included every assessment between the first and final visit) and at the 'final visit'. Pre-treatment disease severity was determined by clinical score on the MGFA Classification [6]. Follow-up assessments were scored using the companion MGFA post-intervention status. These scores were used to assess response to therapy. 'Unchanged', 'worse', 'exacerbation' and 'died of MG' were considered UOs. Among these, 'unchanged' was considered the least severe, and 'died of $\mathrm{MG}$ ' the worst outcome. If a patient was found to have more than one UO during follow-up, only the worst score was considered and associated precipitating factors were statistically analyzed during that time. 'Complete, stable remission', 'pharmacologic remission', 'minimal manifestations', and 'improved' post-intervention status were all considered satisfactory outcomes.

\section{Treatment}

Patient treatment regimes were stratified and recorded with the MGFA Therapy Status classification. Therapeutic
Fig. 1 Treatment regimen and rate of unsatisfactory outcome correlated with care provider group. $* *$ Statistical significance $(P=0.01)$ of the difference between unsatisfactory outcome rates in the two patient groups during follow-up. Immunosuppressant refers to treatment with azathioprine, mycophenolate mofetil or cyclosporine

\begin{tabular}{|c|c|c|}
\hline \multirow[b]{2}{*}{ Type of follow-up, n } & $\begin{array}{c}\text { MG patients } \\
n=41\end{array}$ & nts \\
\hline & neurologists, 15 & neurologists, 26 \\
\hline Mestinon as single therapy, $\mathrm{n}(\%)$ & 0 & $8(31)$ \\
\hline Mestinon \& prednisone, n (\%) & 0 & $1(4)$ \\
\hline Mestinon \& immunosuppressant, n (\%) & $1(7)$ & $2(8)$ \\
\hline Mestinon \& prednisone \& immunosuppressant, n (\%) & $14(93)$ & $15(58)$ \\
\hline Mean delay to prednisone start & 10 days & 300 days \\
\hline Mean delay to immunosupressant start & 21 days & 540 days \\
\hline Intravenous immunoglobulins, n (\%) & $3(20)$ & $9(35)$ \\
\hline Plasma exchange, n (\%) & 0 & $4(15)$ \\
\hline Unsatisfactory outcome, follow-up**, n (\%) & $4(27)$ & $18(69)$ \\
\hline Unsatisfactory outcome, last visit, n (\%) & $1(7)$ & 7 (27) \\
\hline
\end{tabular}


complications were defined as those directly related to any form of specific therapy, such as drug use, operations or radiotherapy. These were reported as adverse effects (AEs). The MGFA morbidity and mortality classification considers infections separately, even if triggered by therapeutic agents. Drug complications were considered related to treatment if they developed during the treatment period, resolved after drug discontinuation or dosage adjustment, and had no other explanation.

\section{Statistical analysis}

Data were analyzed for the study end point and for causes of UOs. We specified interactions a priori, assuming they could influence UOs during follow-up or at the final assessment. The main variables studied were: age at onset, clinical severity at onset (MGFA Clinical Classification), associated co-morbidities, presence and type of antibodies (against AChR or MuSK), electromyographic results, presence of thymoma, delay between first symptoms and beginning of treatment, every independent or associated treatment and the level of training of care providers. The influence of UOs during follow-up on UOs at the last assessment was also analyzed. Data were analyzed with the Fisher's exact test. Significance was set at $P<0.05$. Descriptive statistics with presentation of percentages were used according to the type of variable measured, in particular when subgroups were small or unbalanced. Analyses were conducted using STATA 10.1 software (StataCorp LP, Texas, USA).

\section{Results}

Patient characteristics

Forty one patients were included in the study - the main clinical features and types of follow-up are shown in Table 1. Age at onset was distributed bimodally in females (with modes in the second and eighth decades), and
Table 1 Characteristics of the MG cohort

Data marked with asterisks refer to mean $\pm \mathrm{SD}$ (range) and other data are expressed as number $(n)$ or percent $(\%)$. Single fiber EMG was performed in extensor digitorum communis muscle

$A C h R$ acetylcholine receptor, $E M G$ electromyography, $M G F A$ Myasthenia Gravis Foundation of America, MGFA CC MGFA Clinical Classification, $M u S K$ muscle specific kinase, $R N S$ abnormal repetitive nerve stimulation of at least two different nerves (facial; accessory, radial; oromandibular complex)

\begin{tabular}{|c|c|c|c|}
\hline & $\begin{array}{l}\text { All patients } \\
(n=41)\end{array}$ & $\begin{array}{l}\text { Patients treated by } \\
\text { neuromuscular } \\
\text { neurologists }(n=15)\end{array}$ & $\begin{array}{l}\text { Patients treated } \\
\text { by other neurologists } \\
(n=26)\end{array}$ \\
\hline \multicolumn{4}{|l|}{ Clinical } \\
\hline Sex (men:women) & $21: 20$ & $9: 6$ & $12: 14$ \\
\hline Age at onset (year)* & $54.0 \pm 23.7(6-86)$ & $64.3 \pm 18.2(20-86)$ & $48.1 \pm 24.8(6-85)$ \\
\hline MGFA CC I & $7(17)$ & $2(13)$ & $5(20)$ \\
\hline IIa & $7(17)$ & $3(20)$ & $4(15)$ \\
\hline IIb & 7 (17) & $2(13)$ & $5(20)$ \\
\hline IIIa & $5(12)$ & $1(7)$ & $4(15)$ \\
\hline IIIb & $10(25)$ & $6(40)$ & $4(15)$ \\
\hline $\mathrm{IVb}$ & $5(12)$ & $1(7)$ & $4(15)$ \\
\hline $\mathrm{V}$ & $0(0)$ & $0(0)$ & $0(0)$ \\
\hline Thymoma & $6(15)$ & $3(20)$ & $3(11)$ \\
\hline Follow-up time (months)* & $67.9 \pm 82.4(6-384)$ & $19.9 \pm 17.8(2-60)$ & $95.5 \pm 92.2(6-384)$ \\
\hline \multicolumn{4}{|l|}{ Electrodiagnostic data } \\
\hline RNS study & $38(93)$ & $14(93)$ & $24(92)$ \\
\hline Abnormal & $29(76)$ & $9(64)$ & $20(83)$ \\
\hline Normal & $9(24)$ & $5(36)$ & $4(17)$ \\
\hline Single fiber EMG & $29(76)$ & $14(100)$ & $15(63)$ \\
\hline Abnormal & $20(69)$ & $9(64)$ & $11(73)$ \\
\hline Normal & $9(31)$ & $5(36)$ & $4(27)$ \\
\hline \multicolumn{4}{|l|}{ Laboratory data } \\
\hline AChR antibodies & $41(100)$ & $15(100)$ & $26(100)$ \\
\hline Positive & $36(88)$ & $15(100)$ & $21(81)$ \\
\hline Negative & $5(12)$ & $0(0)$ & $5(19)$ \\
\hline MuSK antibodies & $5(12)$ & $0(0)$ & $5(19)$ \\
\hline Positive & $2(5)$ & $0(0)$ & $2(8)$ \\
\hline Negative & $3(7)$ & $0(0)$ & $3(11)$ \\
\hline
\end{tabular}


unimodally in males, peaking in the sixth decade. RNS was performed in 38 patients (two refused and one could not be performed for technical reasons). Single fiber EMG study could be performed in 29 patients and was abnormal in $69 \%$ of those patients. In nine the RNS and single fiber EMG findings were normal but these patients had positive anti-AChR antibodies.

\section{Therapeutic modalities}

Once diagnosis was established, all patients were immediately treated with pyridostigmine, with or without prednisone, azathioprine or other immunosuppressants. Some received intravenous immunoglobulins (IVIg), plasma exchange (PEx), or underwent thymectomy and radiotherapy. The mean delay from onset of symptoms to treatment was 13.4 months (SD 23.5, range 0-111). Twenty percent received pyridostigmine as monotherapy, $2 \%$ pyridostigmine and prednisone, and $7 \%$ pyridostigmine and azathioprine. The combination of pyridostigmine with prednisone (progressive doses up to $0.75 \mathrm{mg} / \mathrm{kg}$ per day) and azathioprine $(50 \mathrm{mg} /$ day and then up to $2.5 \mathrm{mg} / \mathrm{kg}$ per day), mycophenolate mofetil $(500 \mathrm{mg} /$ day and then up to $2 \mathrm{~g} /$ day) or cyclosporine $(5 \mathrm{mg} / \mathrm{kg}$ per day and then related to serum levels) was used, respectively, in 66,3 and $2 \%$ of our patients. Five PEx were given in each of four patients with severe MGFA post-intervention status. IVIg ( $2 \mathrm{~g} / \mathrm{kg}$ over 5 days) was administrated to 12 patients. Seventeen patients underwent thymectomy. Histological diagnosis in these was thymoma (6/17 patients), hyperplasia (7/17), involution (3/17), and normal (1/17). Two of the operated thymoma patients had associated radiotherapy. Due to the diversity of treatment combinations in such a small patient cohort, statistical analysis of treatment effects was not possible. The differences in therapeutic approaches in those managed by NM neurologists and other neurologists are shown in Fig. 1.

Therapeutic complications

Taken together, independent of any associated UOs, infectious and therapy-specific complications (AEs) occurred in 18/41 patients (44\%). Drug related infections and hepatic, hematologic, metabolic, ocular, cardiac, osteoarticular and digestive AEs to prednisone and azathioprine or to other immunosuppressants were observed in 16 patients (six had more than one); 2 patients suffered from surgical complications, with vena cava replacement needing life-long oral anticoagulation and phrenic nerve injury. The proportion of patients with therapeutic complications due to azathioprine, prednisone, plasma exchange, thymectomy and radiotherapy was, respectively, 33 (10/30), 27 (8/30), 25 (1/4), $12(2 / 17)$ and 50\% (1/2);
IVIg had none $(0 / 12)$. Therapeutic complications were more frequently observed in those patients followed by NM specialists $(53 \%$ compared to 38 in the non-specialist group).

\section{Outcome measures}

The UO rate in the whole cohort reached $54 \%(22 / 41)$ during follow-up and was $20 \%(8 / 41)$ at the final assessment varying significantly between the NM neurologists and the other care groups (Fig. 1). No patient died of MG. Outcome during follow-up was significantly better $(P=0.01)$ when NM neurologists took care of patients. UOs during follow-up were significantly $(P=0.004)$ correlated with the UO rate at final assessment, implying that experiencing a UO during follow-up was predictive of a poor final outcome. It was notable that age at onset, severity of clinical manifestations, associated co-morbidity, presence of thymoma, abnormal EMG results, presence and type of antibodies, longer elapsed time from first symptoms to treatment ( $>3$ months) and every independent or associated treatment failed to show any statistically significant correlation with UO rate during follow-up or at final assessment.

Among all UOs during follow-up, 41\% (9/22) were associated with undertreatment, $23 \%$ (5/22) with poor treatment compliance, another $23 \%$ with infectious complications and $13 \%(3 / 22)$ with serious drug AEs such as toxic hepatitis or intolerance to azathioprine. Nine patients had UOs because of undertreatment: five, though seriously weak, were treated with pyridostigmine alone (IIIb MGFA Clinical Classification or worse); one showed a severely fluctuating clinical score while treated by low dose azathioprine alone; three patients treated with a combination of pyridostigmine, prednisone, and azathioprine had doses prematurely tapered despite ongoing muscle weakness. Two were followed by NM neurologists and seven by other neurologists.

\section{Discussion}

This prospective observational study of 41 treated MG patients enabled us to identify and assess the frequency and the causes of unsatisfactory outcomes (UOs) in treated MG patients during follow-up. We also found that patients were more likely to do better if under the care of an NM neurologist.

Analysis of each UO during follow-up showed that undertreatment, poor treatment compliance, infectious complications and drug-induced AEs were causal factors leading to worse outcome. Undertreatment was the principal cause of UO, with a rate of $41 \%$. Features included 
too-low dose of prednisone, no trial of IVIG or plasma exchange and premature tapering of immunomodulation in the presence of evident ongoing muscle weakness. The common feature is inadequate immune modulation, whatever the treatment used [3]. Treatment modalities varied between physicians: a combination of prednisone and azathioprine or other immunosuppressant was used by NM neurologists in $93 \%$ of their cases, versus $58 \%$ of those patients treated by others. A significantly better outcome in those treated by NM specialists, supports the notion that a combined prednisone-immunosuppressant regimen is effective in treating moderate to severe MG. Poor compliance is a common medical concern [9]. The effects on clinical outcome have been studied in many acute and chronic disorders, but have been rarely assessed in MG. Two studies have shown that remission from MG is related to the confidence of patients in their physician [2, 7]. In our study, poor compliance was responsible for $23 \%$ of all UOs and was only encountered in patients with non-specialist physicians. Possible explanations as to why compliance was improved by having a NM specialist include the increased frequency and regularity of visits and a greater experience with treating MG patients. Similar findings were reported from specialist Myasthenia Gravis Clinics immediately after the discovery of anticholinesterases [8]. Infectious complications and drug-induced AEs were a major cause of UOs, responsible for $36 \%$ of the total. Infections and AEs in our cohort appeared to be related to prednisone and azathioprine use. Prednisone complications (27\% in our study) were less frequent than previously reported by others [11]. Part of this lower complication rate may be due to the systematic use of proton pump inhibitors, calcium and vitamin D3 supplements and regular testing of fasting glucose levels to prevent corticosteroidinduced complications. The azathioprine complication rate we found was $33 \%$, twice than published by some authors [11], but similar to others [5], and probably related to the relatively high doses of azathioprine that we used $(2.5 \mathrm{mg} /$ $\mathrm{kg}$ per day).

Our study revealed that, independently of clinical severity at onset, care by NM neurologists generated significantly better outcome during follow-up $(P=0.01)$ than that achieved by other physicians, and therefore that care provision should be considered a prognostic factor in the management of MG. To the best of our knowledge, such an influence on the outcome of MG has not been reported thus far. It provides empirical evidence that NM subspecialty care plays a beneficial role in outcome which agrees with results recently published by Hill and Ben-Shlomo [4] who reported a crude $69 \%$ reduction in mortality risk when MG patients were cared for by neurologists compared to other hospital physicians. In this study, the authors were unable to determine whether this was because of better management per se or because neurologists were usually based in specialist centers and may have better intensive care support, or both. Alternatively, there may be an element of selection bias so that neurologists cared for less seriously ill patients. In our study, the benefit appears to be due to better management because the rates of undertreatment, poor compliance, infectious complications and drug-induced AEs were different in the two treated groups. In an analogous manner to the demonstration that NM subspecialty training is useful in accurately predicting the likely cause of a peripheral neuropathy [13], it appears that such specialist training is also important in the management of MG.

\section{Conclusion}

Myasthenia gravis remains a challenging field, with a high rate of UOs that are too often due to undertreatment, poor compliance, infections and drug AEs. As about two-thirds of the causes of UOs were due to potentially reversible factors, our study supports the idea that there remains more to be done in optimizing the management of MG, possibly by organizing specialist treatment for all.

Acknowledgments We thank our in-house colleagues and the corresponding physicians who collaborated to the study. This article was reviewed by Richard Frackowiak, chairman of the Lausanne Neurology service.

\section{References}

1. AAEM Quality Assurance Committee (2001) Literature review of the usefulness of repetitive nerve stimulation and single fiber EMG in the electrodiagnostic evaluation of patients with suspected myasthenia gravis or Lambert-Eaton myasthenic syndrome. Muscle Nerve 24:1239-1247

2. Glucker H, Nix WA, Willenberg H, Hoffmann SO (1998) Zur Krankheitsverarbeitung bei Myasthenia gravis im Vergleich mit anderen chronischen neuromuskularer Erkrankungen. Nervenarzt 69:858-863

3. Hart IK, Sathasivam S, Sharshar T (2007) Immunosuppressive agents for myasthenia gravis. Cochrane Database Syst Rev CD005224

4. Hill M, Ben-Shlomo Y (2008) Neurological care and risk of hospital mortality for patients with myasthenia gravis in England. J Neurol Neurosurg Psychiatry 79:421-425

5. Hohlfeld R, Michels M, Heininger K, Besinger U, Toyka KV (1988) Azathioprine toxicity during long-term immunosuppression of generalized myasthenia gravis. Neurology 38:258-261

6. Jaretzki A III, Barohn RJ, Ernstoff RM, Kaminski HJ, Keesey JC, Penn AS, Sanders DB (2000) Myasthenia gravis: recommendations for clinical research standards. Task Force of the Medical Scientific Advisory Board of the Myasthenia Gravis Foundation of America. Neurology 55:16-23

7. Kohler W (2007) Psychosocial aspects in patients with myasthenia gravis. J Neurol 254:II90-II92

8. Lee MR (2007) The miracle at St Alfege's: seventy years on. J R Soc Med 100:108-109 
9. Osterberg L, Blaschke T (2005) Adherence to Medication. N Engl J Med 353:487-497

10. Richman DP, Agius MA (2003) Treatment of autoimmune myasthenia gravis. Neurology 61:1652-1661

11. Rozsa C, Lovas G, Fornadi L, Szabo G, Komoly S (2006) Safety of long-term combined immunosuppressive treatment in myasthenia gravis - analysis of adverse effects of 163 patients. Eur J Neurol 13:947-952
12. Skeie GO, Apostolski S, Evoli A, Gilhus NE, Hart IK, Harms L, Hilton-Jones D, Melms A, Verschuuren J, Horge HW (2006) Guidelines for the treatment of autoimmune neuromuscular transmission disorders. Eur J Neurol 13:691-699

13. Suarez GA, Chalk CH, Russell JW, Kim SM, O'Brien PC, Dyck PJ (2001) Diagnostic accuracy and certainty from sequential evaluations in peripheral neuropathy. Neurology 57:1118-1120 